Brit. Heart F., 1966, 28, 32.

\title{
Effect of Different Degrees of Tilt on Cardiac Output, Heart Rate, and Blood Pressure in Normal Man
}

\author{
JOHN TUCKMAN^ AND JOHN SHILLINGFORD \\ From the Medical Research Council Cardiovascular Research Group, Department of Medicine, Postgraduate Medical \\ School of London, London W.12
}

Many workers have shown that the cardiac output in normal man is less in the upright than in the recumbent position (McMichael and SharpeySchafer, 1944; Stead et al., 1945; Coe, Best, and Lawson, 1950; Wang, Marshall, and Shepherd, 1960; Chapman, Fisher, and Sproule, 1960; Reeves et al., 1961). The physiological events underlying this change are complex and depend on many variables, such as the angle of tilt and length of time the patient remains at a given angle and whether the change is from recumbency to an upright position or vice versa. Posture and its relation to cardiac output here becomes increasingly important with the introduction of hypotensive drugs. The purpose of this study was to determine the relation of the angle of tilting to the changes in cardiac output, blood pressure, and pulse rate in normal man.

\section{SUBJECTS AND METHODS}

Studies were carried out on 33 subjects : 28 women and 5 men, whose average age was 37 years.

The observations were all made three hours after breakfast or lunch. The relative cardiac outputs were measured by the indicator dilution method from dye curves recorded by a photoelectric earpiece (Gabe, Tuckman, and Shillingford, 1962), chopper amplifier, and direct writer recording system. All subjects rested quietly in the supine position for $\mathbf{3 0}$ minutes before the first cardiac output determination. Coomassie blue dye was injected from a calibrated syringe through a three-way tap and eighteen-gauge needle into an antecubital vein, a 5 per cent dextrose-in-water drip keeping the system open between injections. In any one subject the injections of dye were of equal quantity and varied from 20 to $30 \mathrm{mg}$./injection in different subjects.

Received June 1, 1965.

* Post-Doctoral Fellow of the U.S. Public Health Service National Heart Institute.
The total dose to any subject did not exceed $5 \mathrm{mg} . / \mathrm{kg}$. body weight.

Where possible, 9 relative cardiac output determinations were made at intervals of 7 minutes; 3 were made at recumbency, 3 at a given angle of head-up tilt, and 3 after return to recumbency. The tilt positions were $10^{\circ}, 20^{\circ}, 30^{\circ}, 40^{\circ}, 55^{\circ}$, and $60^{\circ}$. Each subject was tilted to only one of these positions. A tilt table with a foot board was used in all procedures. The level of arterial pressure was determined by auscultation.

\section{RESULTS}

Figure 1 shows a representative series of earpiece dye-dilution curves. Since all injections of dye were of equal quantity, the area beneath each (extrapolated) curve is proportional to the reciprocal of the cardiac output. In the example shown here, the increase of area at the tilt position indicates that the cardiac output has fallen 31 per cent compared with the average of the control values.

The hæmodynamic changes associated with tilting to either $10^{\circ}, 20^{\circ}, 30^{\circ}$, or to between 40 and $60^{\circ}$, are shown, respectively, in Tables I, II, III, and IV. In each investigation the measurement of the changes of these variables during tilting was made by comparing the averages of the pre-tilt control and tilt values; this was possible because the variables did not show statistically significant changes within either period.

Cardiac Output. The average cardiac outputs fell at all positions of tilt; $-5,-17,-19$, and -19 per cent at $10^{\circ}, 20^{\circ}, 30^{\circ}$, and $40^{\circ}$ to $60^{\circ}$, respectively; all statistically significant $(p<0.02)$. The changes between $20^{\circ}$ and $60^{\circ}(17 \%-19 \%)$ are significantly greater than the 5 per cent at $10^{\circ}(\mathrm{p}<0.01)$. These results are shown in Fig. 2.

In addition, on return to the flat position, there 


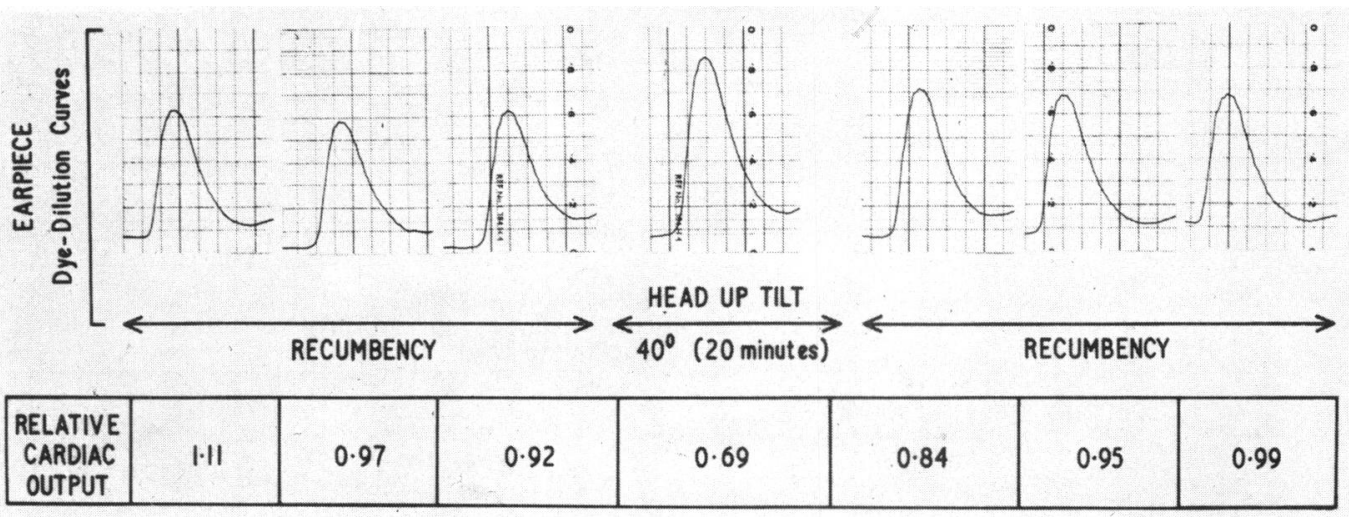

FIG. 1.-Representative series of earpiece dye-dilution curves with relative values of cardiac output below.

TABLE I

CHANGES OF CARDIAC OUTPUT, HEART RATE, AND ARTERIAL PRESSURE AT $10^{\circ}$ OF HEAD-UP TILT

\begin{tabular}{|c|c|c|c|c|c|c|c|c|c|c|c|c|}
\hline \multirow{2}{*}{$\begin{array}{c}\text { Subject and age } \\
(\mathrm{yr} .)\end{array}$} & & \multicolumn{4}{|c|}{ Recumbency } & \multicolumn{4}{|c|}{$10^{\circ}$ Tilt } & \multirow{2}{*}{$\begin{array}{c}\text { Per cent } \\
\text { change }\end{array}$} & \multirow{2}{*}{\multicolumn{2}{|c|}{ Recumbency }} \\
\hline & & & & & Mean & & & & Mean & & & \\
\hline C.H., 53 & $\begin{array}{l}\text { C.O. } \\
\text { H.R. } \\
\text { B.P. } \\
\text { Time }\end{array}$ & $\begin{array}{l}0.94 \\
64 \\
120 / 80\end{array}$ & $\begin{array}{l}1 \cdot 06 \\
62 \\
120 / 75\end{array}$ & & $\begin{array}{l}1 \cdot 00 \\
63 \\
120 / 78\end{array}$ & & & $\begin{array}{l}0.99 \\
62 \\
120 / 85 \\
22^{\prime}\end{array}$ & $\begin{array}{l}0.99 \\
62 \\
120 / 85\end{array}$ & -1 & & $\begin{array}{l}1.06 \\
62 \\
120 / 75 \\
13^{\prime}\end{array}$ \\
\hline D.C., 40 & $\begin{array}{l}\text { C.O. } \\
\text { H.R. } \\
\text { B.P. } \\
\text { Time }\end{array}$ & $\begin{array}{l}1 \cdot 30 \\
70 \\
140 / 90\end{array}$ & $\begin{array}{l}0.99 \\
74 \\
145 / 90\end{array}$ & $\begin{array}{l}0.98 \\
70 \\
135 / 85\end{array}$ & $\begin{array}{l}1 \cdot 00 \\
71 \\
140 / 88\end{array}$ & $\begin{array}{l}0.93 \\
72 \\
135 / 85 \\
9^{\prime} \\
\end{array}$ & $\begin{array}{l}0.95 \\
72 \\
140 / 90 \\
17^{\prime} \\
\end{array}$ & $\begin{array}{l}1 \cdot 04 \\
72 \\
140 / 90 \\
24^{\prime} \\
\end{array}$ & $\begin{array}{l}0.97 \\
72 \\
138 / 88\end{array}$ & -3 & & $\begin{array}{l}1 \cdot 02 \\
70 \\
145 / 90 \\
12^{\prime}\end{array}$ \\
\hline D.S., 29 & $\begin{array}{l}\text { C.O. } \\
\text { H.R. } \\
\text { B.P. } \\
\text { Time }\end{array}$ & $\begin{array}{l}9 \cdot 99 \\
66 \\
105 / 65\end{array}$ & $\begin{array}{l}0.92 \\
70 \\
100 / 65\end{array}$ & $\begin{array}{l}1 \cdot 10 \\
62 \\
100 / 65\end{array}$ & $\begin{array}{l}1 \cdot 00 \\
66 \\
102 / 65\end{array}$ & & $\begin{array}{l}0.96 \\
66 \\
90 / 60 \\
11^{\prime} \\
\end{array}$ & $\begin{array}{l}0.94 \\
66 \\
110 / 75 \\
20^{\prime} \\
\end{array}$ & $\begin{array}{l}0.95 \\
66 \\
100 / 68\end{array}$ & -5 & & \\
\hline N.W., 28 & $\begin{array}{l}\text { C.O. } \\
\text { H.R. } \\
\text { B.P. } \\
\text { Time }\end{array}$ & $\begin{array}{l}0.95 \\
78 \\
150 / 75\end{array}$ & $\begin{array}{l}1 \cdot 00 \\
81 \\
150 / 75\end{array}$ & $\begin{array}{l}1.05 \\
77 \\
150 / 70\end{array}$ & $\begin{array}{l}1 \cdot 00 \\
79 \\
150 / 73\end{array}$ & $\begin{array}{l}0 \cdot 85 \\
84 \\
150 / 85 \\
7^{\prime}\end{array}$ & $\begin{array}{l}9 \cdot 88 \\
16^{\prime}\end{array}$ & $\begin{array}{l}0 \cdot 87 \\
77 \\
150 / 90 \\
23^{\prime}\end{array}$ & $\begin{array}{l}0 \cdot 87 \\
80 \\
150 / 88\end{array}$ & -13 & $\begin{array}{l}0 \cdot 88 \\
84 \\
150 / 80 \\
7^{\prime}\end{array}$ & $\begin{array}{l}0.98 \\
81 \\
145 / 80 \\
13^{\prime}\end{array}$ \\
\hline V.H., 45 & $\begin{array}{l}\text { C.O. } \\
\text { H.R. } \\
\text { B.P. } \\
\text { Time }\end{array}$ & $\begin{array}{l}1.06 \\
68 \\
95 / 70\end{array}$ & $\begin{array}{l}0.99 \\
66 \\
95 / 70\end{array}$ & $\begin{array}{l}0 \cdot 96 \\
64 \\
95 / 70\end{array}$ & $\begin{array}{l}1 \cdot 00 \\
66 \\
95 / 70\end{array}$ & & $\begin{array}{l}0 \cdot 94 \\
64 \\
90 / 75 \\
23^{\prime} \\
\end{array}$ & $\begin{array}{l}1 \cdot 08 \\
69 \\
95 / 75 \\
29^{\prime} \\
\end{array}$ & $\begin{array}{l}1 \cdot 01 \\
66 \\
92 / 75\end{array}$ & +1 & & \\
\hline E.L., 18 & $\begin{array}{l}\text { C.O. } \\
\text { H.R. } \\
\text { B.P. } \\
\text { Time }\end{array}$ & $\begin{array}{l}1.00 \\
70 \\
100 / 70\end{array}$ & $\begin{array}{l}1 \cdot 01 \\
70 \\
95 / 70\end{array}$ & 0.99 & $\begin{array}{l}1.00 \\
70 \\
98 / 70\end{array}$ & & $\begin{array}{l}0.97 \\
69 \\
115 / 80 \\
13^{\prime} \\
\end{array}$ & $\begin{array}{l}0 \cdot 93 \\
72 \\
105 / 75 \\
20^{\prime} \\
\end{array}$ & $\begin{array}{l}0.95 \\
70 \\
110 / 78\end{array}$ & -5 & $\begin{array}{l}0.96 \\
70 \\
95 / 75 \\
8^{\prime} \\
\end{array}$ & \\
\hline S.M., 29 & $\begin{array}{l}\text { C.O. } \\
\text { H.R. } \\
\text { B.P. } \\
\text { Time }\end{array}$ & $\begin{array}{l}1.00 \\
78 \\
140 / 85\end{array}$ & $\begin{array}{l}1 \cdot 01 \\
84 \\
135 / 80\end{array}$ & $\begin{array}{l}0.98 \\
84 \\
140 / 85\end{array}$ & $\begin{array}{l}1 \cdot 00 \\
82 \\
138 / 83\end{array}$ & & $\begin{array}{l}0.93 \\
80 \\
140 / 85 \\
20^{\prime} \\
\end{array}$ & $\begin{array}{l}0.94 \\
80 \\
26^{\prime} \\
\end{array}$ & $\begin{array}{l}0.94 \\
80 \\
140 / 85\end{array}$ & -6 & & \\
\hline I.H., 45 & $\begin{array}{l}\text { C.O. } \\
\text { H.R. } \\
\text { B.P. } \\
\text { Time }\end{array}$ & $\begin{array}{l}0.97 \\
68 \\
140 / 75\end{array}$ & $\begin{array}{l}1.05 \\
66 \\
135 / 70\end{array}$ & $\begin{array}{l}0.97 \\
66 \\
140 / 75\end{array}$ & $\begin{array}{l}1 \cdot 00 \\
67 \\
138 / 73\end{array}$ & & $\begin{array}{l}0.90 \\
60 \\
135 / 85 \\
11^{\prime} \\
\end{array}$ & $\begin{array}{l}0.91 \\
63 \\
135 / 85 \\
19^{\prime} \\
\end{array}$ & $\begin{array}{l}0.90 \\
62 \\
135 / 85\end{array}$ & -10 & $\begin{array}{l}1 \cdot 30 \\
56 \\
135 / 80 \\
10^{\prime} \\
\end{array}$ & $\begin{array}{l}0.99 \\
56 \\
135 / 80 \\
14^{\prime}\end{array}$ \\
\hline Average & $\begin{array}{l}\text { C.O. } \\
\text { H.R. } \\
\text { B.P. }\end{array}$ & & & & $\begin{array}{l}1.00 \\
70 \\
123 / 75\end{array}$ & & & & $\begin{array}{l}0.95 \\
70 \\
123 / 82\end{array}$ & -5 & & \\
\hline
\end{tabular}

C.O. = Cardiac output. To make the changes in cardiac output readily apparent, the cardiac outputs calculated from a series of earpiece dye-dilution curves are expressed relative to the average reciprocal of the areas $\left(\mathrm{cm} .^{-2}\right)$ under the curves of the pre-

H.R. = Heart rate tilt control determinations of the series, and this in turn is expressed as unity.

B.P. = Systemic arterial pressure

Time $=$ Time in minutes (a) after placing the subject in tilt position and (b) after returning the subject to recumbency from the tilt position. * Significant at the $p=0.05$ (or less) level. 
TABLE II

CHANGES OF CARDIAC OUTPUT, HEART RATE, AND ARTERIAL PRESSURE AT $20^{\circ}$ OF HEAD-UP TILT

\begin{tabular}{|c|c|c|c|c|c|c|c|c|c|c|c|c|c|}
\hline \multirow{2}{*}{$\begin{array}{c}\begin{array}{c}\text { Subject } \\
\text { and age } \\
\text { (yr.) }\end{array} \\
\end{array}$} & & \multicolumn{4}{|c|}{ Recumbency } & \multicolumn{4}{|c|}{$20^{\circ}$ Tilt } & \multirow{3}{*}{$\begin{array}{c}\text { Per cent } \\
\text { change }\end{array}$} & \multirow{2}{*}{\multicolumn{3}{|c|}{ Recumbency }} \\
\hline & & & & & Mean & & & & Mean & & & & \\
\hline MM., 16 & $\begin{array}{l}\text { C.O. } \\
\text { H.R. } \\
\text { B.P. } \\
\text { Time }\end{array}$ & $\begin{array}{l}0.99 \\
72 \\
100 / 56\end{array}$ & $\begin{array}{l}0.95 \\
72 \\
96 / 56\end{array}$ & $\begin{array}{l}1 \cdot 06 \\
72 \\
98 / 56\end{array}$ & $\begin{array}{l}1 \cdot 00 \\
72 \\
98 / 56\end{array}$ & $\begin{array}{l}0 \cdot 94 \\
74 \\
96 / 56 \\
7^{\prime}\end{array}$ & $\begin{array}{l}0.90 \\
84 \\
106 / 64 \\
14^{\prime}\end{array}$ & $\begin{array}{l}0 \cdot 85 \\
79 \\
106 / 66 \\
21^{\prime}\end{array}$ & $\begin{array}{l}0 \cdot 90 \\
79 \\
103 / 62\end{array}$ & & $\begin{array}{l}0 \cdot 89 \\
82 \\
100 / 58 \\
7^{\prime}\end{array}$ & $\begin{array}{l}0 \cdot 82 \\
84 \\
104 / 62 \\
14^{\prime}\end{array}$ & $\begin{array}{l}0 \cdot 82 \\
76 \\
100 / 58 \\
21^{\prime}\end{array}$ \\
\hline R.M., 45 & $\begin{array}{l}\text { C.O. } \\
\text { H.R. } \\
\text { B.P. } \\
\text { Time }\end{array}$ & $\begin{array}{l}1 \cdot 16 \\
60 \\
108 / 70\end{array}$ & $\begin{array}{l}0.90 \\
58 \\
106 / 74\end{array}$ & $\begin{array}{l}0.94 \\
60 \\
110 / 76\end{array}$ & $\begin{array}{l}1 \cdot 00 \\
49 \\
108 / 73\end{array}$ & $\begin{array}{l}0 \cdot 82 \\
59 \\
116 / 80 \\
7^{\prime}\end{array}$ & $\begin{array}{l}0 \cdot 82 \\
60 \\
120 / 80 \\
14^{\prime}\end{array}$ & & $\begin{array}{l}0 \cdot 82 \\
60 \\
118 / 80\end{array}$ & -18 & & & \\
\hline A.O., 50 & $\begin{array}{l}\text { C.O. } \\
\text { H.R. } \\
\text { B.P. } \\
\text { Time }\end{array}$ & $\begin{array}{l}1.06 \\
89 \\
155 / 90\end{array}$ & $\begin{array}{l}86 \\
160 / 90\end{array}$ & $\begin{array}{l}0.94 \\
92 \\
160 / 90\end{array}$ & $\begin{array}{l}1 \cdot 00 \\
89 \\
158 / 90\end{array}$ & $\begin{array}{l}0 \cdot 84 \\
89 \\
160 / 90 \\
8^{\prime}\end{array}$ & $\begin{array}{l}0.77 \\
89 \\
15^{\prime}\end{array}$ & & $\begin{array}{l}0.80 \\
89 \\
160 / 90\end{array}$ & -20 & $\begin{array}{l}0 \cdot 92 \\
92 \\
165 / 95 \\
8^{\prime}\end{array}$ & & \\
\hline M.Y., 37 & $\begin{array}{l}\text { C.O. } \\
\text { H.R. } \\
\text { B.P. } \\
\text { Time }\end{array}$ & $\begin{array}{l}1 \cdot 08 \\
74 \\
100 / 58\end{array}$ & $\begin{array}{l}0.90 \\
70 \\
90 / 58\end{array}$ & $\begin{array}{l}1 \cdot 02 \\
69 \\
90 / 58\end{array}$ & $\begin{array}{l}1 \cdot 00 \\
71 \\
93 / 58\end{array}$ & $\begin{array}{l}0 \cdot 77 \\
67 \\
88 / 52 \\
7^{\prime} \\
\end{array}$ & $\begin{array}{l}0.65 \\
68 \\
90 / 56 \\
14^{\prime} \\
\end{array}$ & & $\begin{array}{l}0.71 \\
68 \\
89 / 54\end{array}$ & -29 & $\begin{array}{l}0 \cdot 68 \\
64 \\
90 / 56 \\
7^{\prime}\end{array}$ & & \\
\hline E.M., 45 & $\begin{array}{l}\text { C.O. } \\
\text { H.R. } \\
\text { B.P. } \\
\text { Time } \\
\end{array}$ & $\begin{array}{l}1 \cdot 02 \\
96 \\
110 / 80\end{array}$ & $\begin{array}{l}1 \cdot 04 \\
88 \\
108 / 86\end{array}$ & $\begin{array}{l}0.94 \\
92 \\
108 / 86\end{array}$ & $\begin{array}{l}1 \cdot 00 \\
92 \\
109 / 84\end{array}$ & $\begin{array}{l}0 \cdot 87 \\
80 \\
108 / 80 \\
6^{\prime} \\
\end{array}$ & $\begin{array}{l}0 \cdot 71 \\
84 \\
110 / 84 \\
13^{\prime}\end{array}$ & $\begin{array}{l}0 \cdot 65 \\
84 \\
116 / 84 \\
20^{\prime} \\
\end{array}$ & $\begin{array}{l}0 \cdot 74 \\
83 \\
111 / 83\end{array}$ & -26 & $\begin{array}{l}0 \cdot 72 \\
86 \\
114 / 80 \\
7^{\prime}\end{array}$ & $\begin{array}{l}0 \cdot 61 \\
84 \\
110 / 78 \\
14^{\prime}\end{array}$ & $\begin{array}{l}0 \cdot 74 \\
84 \\
108 / 78 \\
21^{\prime}\end{array}$ \\
\hline S.C., 25 & $\begin{array}{l}\text { C.O. } \\
\text { H.R. } \\
\text { B.P. } \\
\text { Time }\end{array}$ & $\begin{array}{l}1 \cdot 16 \\
78 \\
108 / 68\end{array}$ & $\begin{array}{l}0.92 \\
76 \\
110 / 68\end{array}$ & $\begin{array}{l}0.92 \\
86 \\
116 / 62\end{array}$ & $\begin{array}{l}1 \cdot 00 \\
80 \\
111 / 66\end{array}$ & $\begin{array}{l}0 \cdot 84 \\
72 \\
108 / 68 \\
7^{\prime}\end{array}$ & $\begin{array}{l}0 \cdot 98 \\
78 \\
104 / 68 \\
14^{\prime} \\
\end{array}$ & & $\begin{array}{l}0 \cdot 71 \\
75 \\
106 / 68\end{array}$ & -9 & $\begin{array}{l}0 \cdot 88 \\
76 \\
110 / 70 \\
7^{\prime}\end{array}$ & $\begin{array}{l}0 \cdot 92 \\
78 \\
110 / 70 \\
14^{\prime}\end{array}$ & \\
\hline S.T., 35 & $\begin{array}{l}\text { C.O. } \\
\text { H.R. } \\
\text { B.P. } \\
\text { Time }\end{array}$ & $\begin{array}{l}0.98 \\
58 \\
110 / 80\end{array}$ & $\begin{array}{l}1 \cdot 01 \\
58 \\
105 / 75\end{array}$ & $\begin{array}{l}1 \cdot 00 \\
54 \\
105 / 75\end{array}$ & $\begin{array}{l}1.00 \\
57 \\
107 / 77\end{array}$ & $\begin{array}{l}0 \cdot 90 \\
58 \\
105 / 75 \\
7^{\prime} \\
\end{array}$ & $\begin{array}{l}0 \cdot 96 \\
54 \\
110 / 75 \\
14^{\prime} \\
\end{array}$ & $\begin{array}{l}0 \cdot 88 \\
54 \\
110 / 80 \\
21^{\prime} \\
\end{array}$ & $\begin{array}{l}0 \cdot 91 \\
55 \\
108 / 77\end{array}$ & -9 & $\begin{array}{l}0 \cdot 92 \\
52 \\
110 / 80 \\
7^{\prime}\end{array}$ & $\begin{array}{l}0.92 \\
52 \\
110 / 80 \\
14^{\prime} \\
\end{array}$ & $\begin{array}{l}0 \cdot 93 \\
54 \\
105 / 80 \\
21^{\prime} \\
\end{array}$ \\
\hline I.B., 41 & $\begin{array}{l}\text { C.O. } \\
\text { H.R. } \\
\text { B.P. } \\
\text { Time } \\
\end{array}$ & $\begin{array}{l}0 \cdot 89 \\
68 \\
125 / 90\end{array}$ & $\begin{array}{l}1 \cdot 12 \\
67 \\
136 / 80\end{array}$ & & $\begin{array}{l}1 \cdot 00 \\
68 \\
130 / 85\end{array}$ & $\begin{array}{l}0 \cdot 89 \\
66 \\
110 / 85 \\
10^{\prime} \\
\end{array}$ & & $\begin{array}{l}0 \cdot 85 \\
68 \\
130 / 90 \\
18^{\prime} \\
\end{array}$ & $\begin{array}{l}0 \cdot 87 \\
67 \\
120 / 88\end{array}$ & -13 & & $\begin{array}{l}0 \cdot 89 \\
66 \\
130 / 90 \\
13^{\prime}\end{array}$ & $\begin{array}{l}0.84 \\
20^{\prime}\end{array}$ \\
\hline Average & $\begin{array}{l}\text { C.O. } \\
\text { H.R. } \\
\text { B.P. }\end{array}$ & & & & $\begin{array}{l}1 \cdot 00 \\
74 \\
114 / 74\end{array}$ & & & & $\begin{array}{l}0 \cdot 83 \\
72 \\
114 / 75\end{array}$ & -17 & & & \\
\hline
\end{tabular}

* Significant at the $\mathrm{p}=0.05$ (or less) level.

was a delay in the increase of cardiac output to the pre-tilt control values. Of the 19 subjects who had a decrease of cardiac output of 5 per cent or more when tilted, 17 had sufficient data for analysis in the

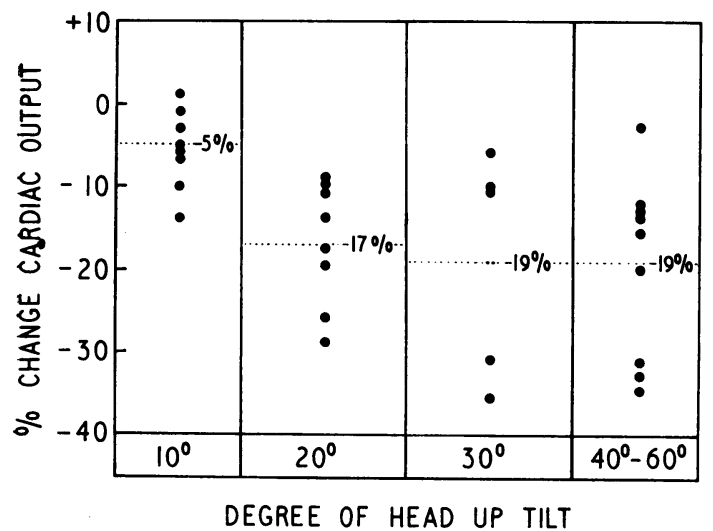

FIG. 2.-Percentage change in cardiac output following different degrees of head-up tilt. post-tilt period. When the data are divided into those from the first 10 minutes after return to recumbency and those from between 11 and 25 minutes after this event, the cardiac outputs are 10 per cent and 13 per cent less, respectively, than the pre-tilt control values $(\mathrm{p}<0.01)$. Data after 25 minutes were insufficient for adequate analysis.

Heart Rate. The heart rate remained essentially unchanged at $10^{\circ}$ and $20^{\circ}$ but it did increase at $30^{\circ}$ $\left(77 / \mathrm{min}\right.$. to $83 / \mathrm{min}$.) and between $40^{\circ}$ and $60^{\circ}$ $(70 / \mathrm{min}$. to $83 / \mathrm{min}$.). These changes were statistically significant $(p<0.05)$ only in the group tilted to between $30^{\circ}$ and $60^{\circ}$. The data relating to changes of heart rate to the degree of tilt are shown in Fig. 3.

Stroke Volume. The average stroke volumes decreased at all angles of tilt; $-4,-15,-24$, and -31 per cent at $10^{\circ}, 20^{\circ}, 30^{\circ}$, and between $40^{\circ}$ and $60^{\circ}$, respectively. These were all statistically significant changes at the $p<0.05$ level and are shown in Fig. 4. 
TABLE III

CHANGES IN CARDIAC OUTPUT, HEART RATE, AND ARTERIAL PRESSURE AT $30^{\circ}$ OF HEAD-UP TILT

\begin{tabular}{|c|c|c|c|c|c|c|c|c|c|c|c|c|}
\hline \multirow{2}{*}{$\begin{array}{c}\text { Subject and age } \\
(y r .)\end{array}$} & & \multicolumn{4}{|c|}{ Recumbency } & \multicolumn{4}{|c|}{$30^{\circ}$ Tilt } & \multirow{2}{*}{$\begin{array}{c}\text { Per cent } \\
\text { change }\end{array}$} & \multirow{2}{*}{\multicolumn{2}{|c|}{ Recumbency }} \\
\hline & & & & & Mean & & & & Mean & & & \\
\hline G.D., 37 & $\begin{array}{l}\text { C.O. } \\
\text { H.R. } \\
\text { B.P. } \\
\text { Time }\end{array}$ & $\begin{array}{l}1 \cdot 03 \\
84 \\
156 / 94\end{array}$ & $\begin{array}{l}0 \cdot 97 \\
80 \\
160 / 96\end{array}$ & $\begin{array}{l}1 \cdot 00 \\
80 \\
156 / 96\end{array}$ & $\begin{array}{l}1 \cdot 00 \\
81 \\
157 / 95\end{array}$ & $\begin{array}{l}0 \cdot 73 \\
90 \\
144 / 88 \\
7^{\prime}\end{array}$ & $\begin{array}{l}0 \cdot 67 \\
83 \\
134 / 78 \\
14^{\prime}\end{array}$ & $\begin{array}{l}0.68 \\
82 \\
140 / 84 \\
22^{\prime}\end{array}$ & $\begin{array}{l}0.69 \\
82 \\
139 / 83\end{array}$ & -31 & $\begin{array}{l}0 \cdot 89 \\
74 \\
144 / 95 \\
7^{\prime}\end{array}$ & $\begin{array}{l}0 \cdot 74 \\
80 \\
144 / 92 \\
14^{\prime}\end{array}$ \\
\hline E.E., 49 & $\begin{array}{l}\text { C.O. } \\
\text { H.R. } \\
\text { B.P. } \\
\text { Time }\end{array}$ & $\begin{array}{l}1 \cdot 10 \\
68 \\
140 / 75\end{array}$ & $\begin{array}{l}0.90 \\
68 \\
135 / 80\end{array}$ & & $\begin{array}{l}1 \cdot 00 \\
68 \\
138 / 78\end{array}$ & & $\begin{array}{l}0 \cdot 80 \\
66 \\
170 / 90 \\
16^{\prime}\end{array}$ & & $\begin{array}{l}0 \cdot 80 \\
66 \\
170 / 90\end{array}$ & -20 & & \\
\hline R.H., 23 & $\begin{array}{l}\text { C.O. } \\
\text { H.R. } \\
\text { B.P. } \\
\text { Time }\end{array}$ & $\begin{array}{l}1 \cdot 03 \\
72 \\
120 / 80\end{array}$ & $\begin{array}{l}0 \cdot 97 \\
75 \\
110 / 80\end{array}$ & & $\begin{array}{l}1 \cdot 00 \\
74 \\
115 / 80\end{array}$ & & $\begin{array}{l}0 \cdot 81 \\
85 \\
11^{\prime}\end{array}$ & & $\begin{array}{l}0 \cdot 81 \\
85\end{array}$ & -19 & & \\
\hline V.E., 42 & $\begin{array}{l}\text { C.O. } \\
\text { H.R. } \\
\text { B.P. } \\
\text { Time }\end{array}$ & $\begin{array}{l}1 \cdot 03 \\
74 \\
130 / 90\end{array}$ & $\begin{array}{l}0 \cdot 97 \\
74 \\
130 / 90\end{array}$ & & $\begin{array}{l}1 \cdot 00 \\
74 \\
130 / 90\end{array}$ & $\begin{array}{l}0 \cdot 94 \\
74 \\
135 / 95 \\
6^{\prime}\end{array}$ & & & $\begin{array}{l}0 \cdot 94 \\
74 \\
135 / 95\end{array}$ & -6 & & \\
\hline P.S., 45 & $\begin{array}{l}\text { C.O. } \\
\text { H.R. } \\
\text { B.P. } \\
\text { Time }\end{array}$ & $\begin{array}{l}9 \cdot 89 \\
100 \\
130 / 85\end{array}$ & $1 \cdot 17$ & $\begin{array}{l}0.94 \\
103 \\
120 / 90\end{array}$ & $\begin{array}{l}1 \cdot 00 \\
102 \\
125 / 88\end{array}$ & $\begin{array}{l}0.66 \\
106 \\
135 / 90 \\
10^{\prime}\end{array}$ & $\begin{array}{l}0.63 \\
106 \\
16^{\prime}\end{array}$ & & $\begin{array}{l}0.64 \\
106 \\
135 / 90\end{array}$ & -36 & & \\
\hline E.G. & $\begin{array}{l}\text { C.O. } \\
\text { H.R. } \\
\text { B.P. }\end{array}$ & $\begin{array}{l}0.87 \\
72 \\
115 / 90\end{array}$ & $\begin{array}{l}1 \cdot 13 \\
74 \\
115 / 90\end{array}$ & & $\begin{array}{l}1 \cdot 00 \\
73 \\
115 / 90\end{array}$ & $\begin{array}{l}0 \cdot 82 \\
90 \\
125 / 100\end{array}$ & & & $\begin{array}{l}0 \cdot 82 \\
90 \\
125 / 100\end{array}$ & -18 & & \\
\hline S.R., 29 & $\begin{array}{l}\text { C.O. } \\
\text { H.R. } \\
\text { B.P. } \\
\text { Time }\end{array}$ & $\begin{array}{l}0.95 \\
75 \\
110 / 70\end{array}$ & $\begin{array}{l}1 \cdot 05 \\
84 \\
110 / 70\end{array}$ & & $\begin{array}{l}1 \cdot 00 \\
80 \\
110 / 70\end{array}$ & & $\begin{array}{l}0 \cdot 88 \\
96 \\
120 / 90 \\
12^{\prime}\end{array}$ & $\begin{array}{l}0.92 \\
92 \\
120 / 90 \\
23^{\prime}\end{array}$ & $\begin{array}{l}0 \cdot 90 \\
94 \\
120 / 90\end{array}$ & -10 & & \\
\hline H.W., 71 & $\begin{array}{l}\text { C.O. } \\
\text { H.R. } \\
\text { B.P. } \\
\text { Time }\end{array}$ & $\begin{array}{l}1 \cdot 08 \\
64 \\
155 / 80\end{array}$ & $\begin{array}{l}0 \cdot 92 \\
66 \\
155 / 80\end{array}$ & & $\begin{array}{l}1 \cdot 00 \\
65 \\
155 / 80\end{array}$ & $\begin{array}{l}0 \cdot 93 \\
68 \\
6^{\prime}\end{array}$ & $\begin{array}{l}0 \cdot 88 \\
68 \\
160 / 100 \\
11^{\prime}\end{array}$ & & $\begin{array}{l}0 \cdot 90 \\
68 \\
160 / 100\end{array}$ & -10 & & \\
\hline Average & $\begin{array}{l}\text { C.O. } \\
\text { H.R. } \\
\text { B.P. }\end{array}$ & & & & $\begin{array}{l}1 \cdot 00 \\
77 \\
133 / 84\end{array}$ & & & & $\begin{array}{l}0 \cdot 81 \\
83 \\
141 / 93\end{array}$ & $-19 \star$ & & \\
\hline
\end{tabular}

$\star$ Significant at the $p=0.05$ (or less) level.

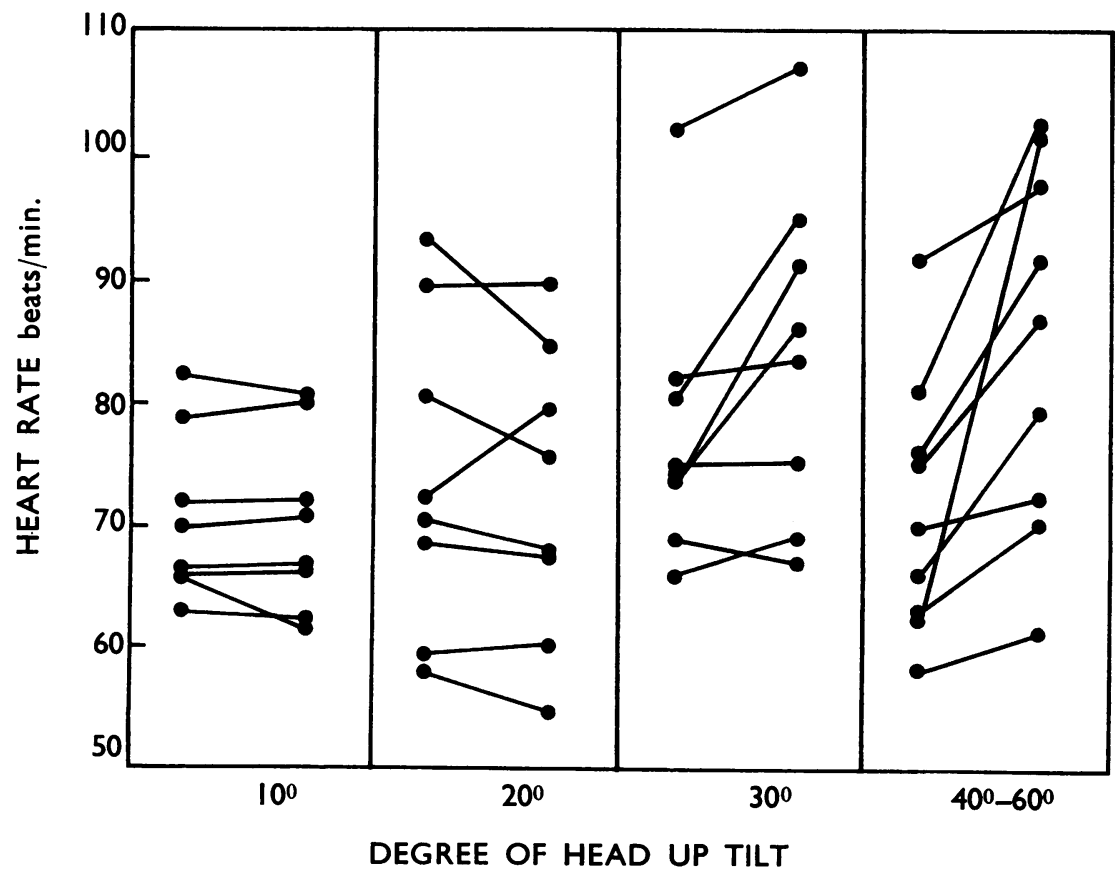

FIG. 3.-Change in heart rate following different degrees of head-up tilt. 
TABLE IV

CHANGES IN CARDIAC OUTPUT, HEART RATE, AND ARTERIAL PRESSURE AT $40^{\circ}-60^{\circ}$ OF HEAD-UP TILT

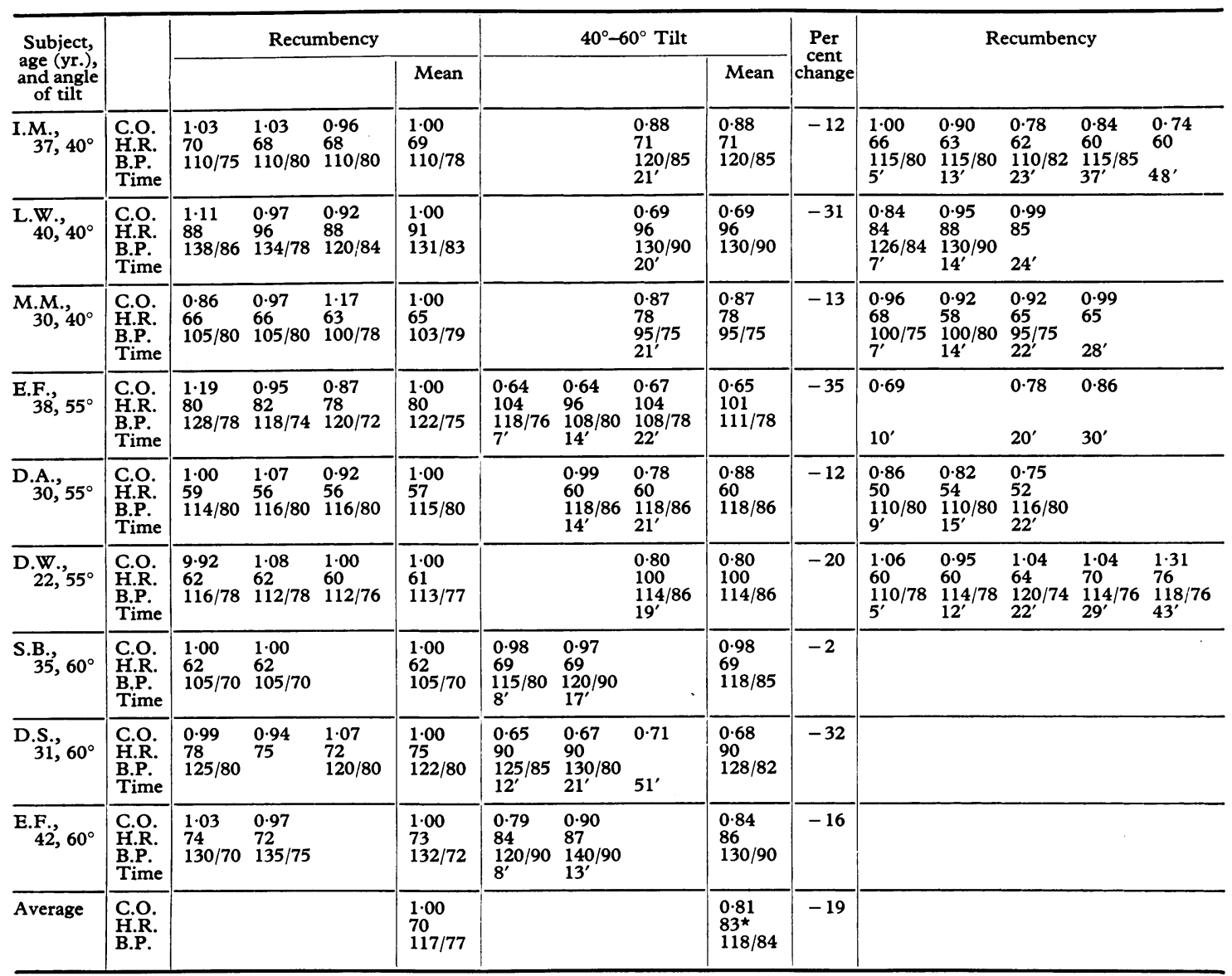

* Significant at $\mathrm{p}=0.05$ (or less) level.

Arterial Pressures. (a) Diastolic pressures increased at $10^{\circ}$ (75 to $82 \mathrm{~mm}$. $\mathrm{Hg}$ ), $30^{\circ}$ (84 to $93 \mathrm{~mm}$. $\mathrm{Hg}$ ), and between $40^{\circ}-60^{\circ}$ (77 to $84 \mathrm{~mm}$. $\mathrm{Hg}$ ). The changes were first short of statistical significance at $10^{\circ}$ but were significant at $30^{\circ}$ and between $40^{\circ}$ and $60^{\circ} \quad(\mathrm{p}<0.05$ and $\mathrm{p}<0.01$ respectively). These results are shown in Fig. 5.

(b) Systolic pressures did not change significantly at any of the tilt positions.

(c) Pulse pressures did not alter significantly at any angle of tilt but decreased at $10^{\circ}$ ( 48 to $42 \mathrm{~mm}$. $\mathrm{Hg}$ ) and between $40^{\circ}$ and $60^{\circ}$ (40 to $34 \mathrm{~mm}$. $\mathrm{Hg}$ ).

(d) Mean arterial pressures were estimated by adding one-third of the pulse pressures to the diastolic pressures. The mean arterial pressure did not change significantly at any tilt position; however, it did increase at $10^{\circ}$ (19 to $86 \mathrm{~mm} . \mathrm{Hg}$ ), $30^{\circ}$ (100 to $109 \mathrm{~mm}$. $\mathrm{Hg}$ ), and between $40^{\circ}$ and $60^{\circ}$ (91 to 95 $\mathrm{mm} . \mathrm{Hg}$ ).

Peripheral Resistance. It can be shown that the percentage change in peripheral resistance in each study is $100\left(\frac{P_{2} \cdot \text { C.O. }}{P_{1} \cdot \text { C.O.2 }}-1\right)$, where $P_{2}$ and $P_{1}$ are the mean arterial pressure and C.O. ${ }_{1}$ and C.O. 2 are the corresponding cardiac outputs. It will be seen that the absolute values for cardiac outputs are not required. Calculated in this way peripheral resistance increased at all angles of tilt: $+11,+18,+35$, and +30 per cent at $10^{\circ}, 20^{\circ}, 30^{\circ}$, and between $40^{\circ}$ and $60^{\circ}$, respectively (Fig. 6). These changes were all significant $(p<0.05)$. 
Effect of Tilt on Cardiac Output, Heart Rate, and Blood Pressure

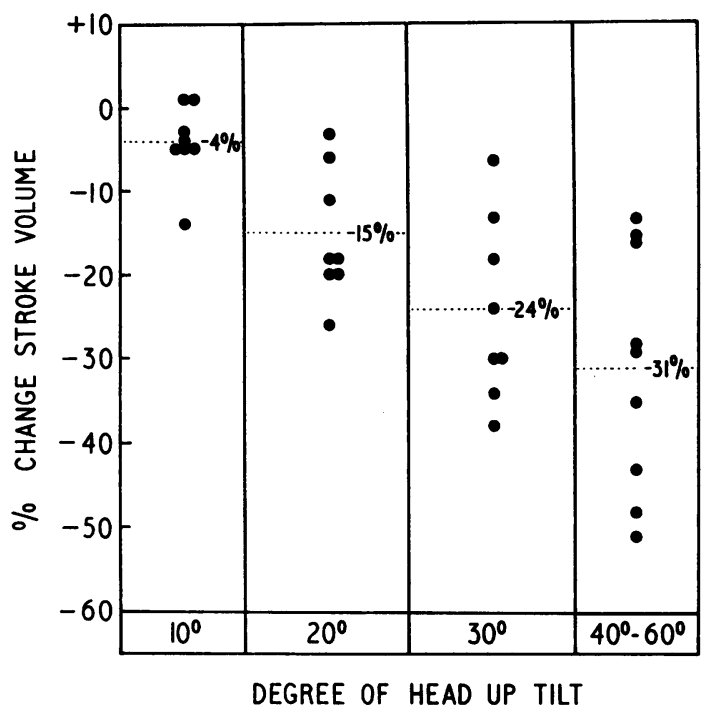

Fig. 4.-Percentage change in stroke volume following different degrees of head-up tilt.

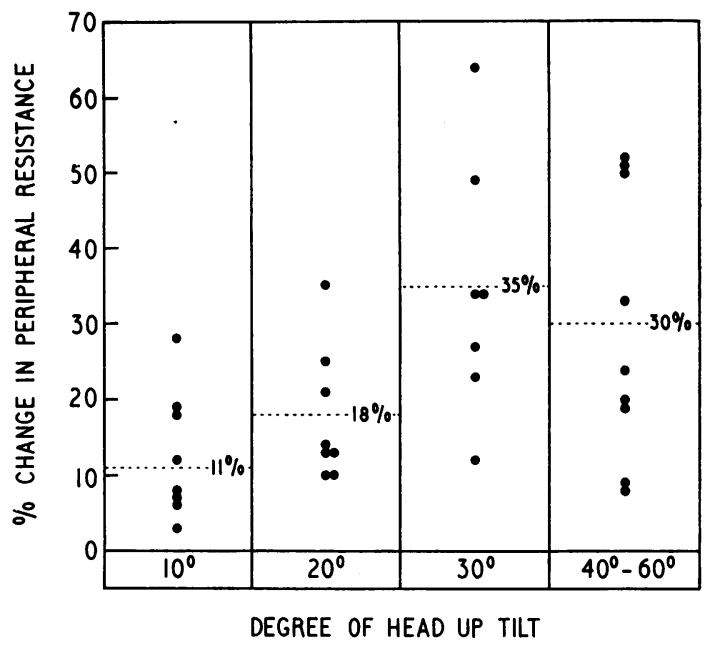

FIG. 6.-Percentage change in peripheral resistance following different degrees of head-up tilt.

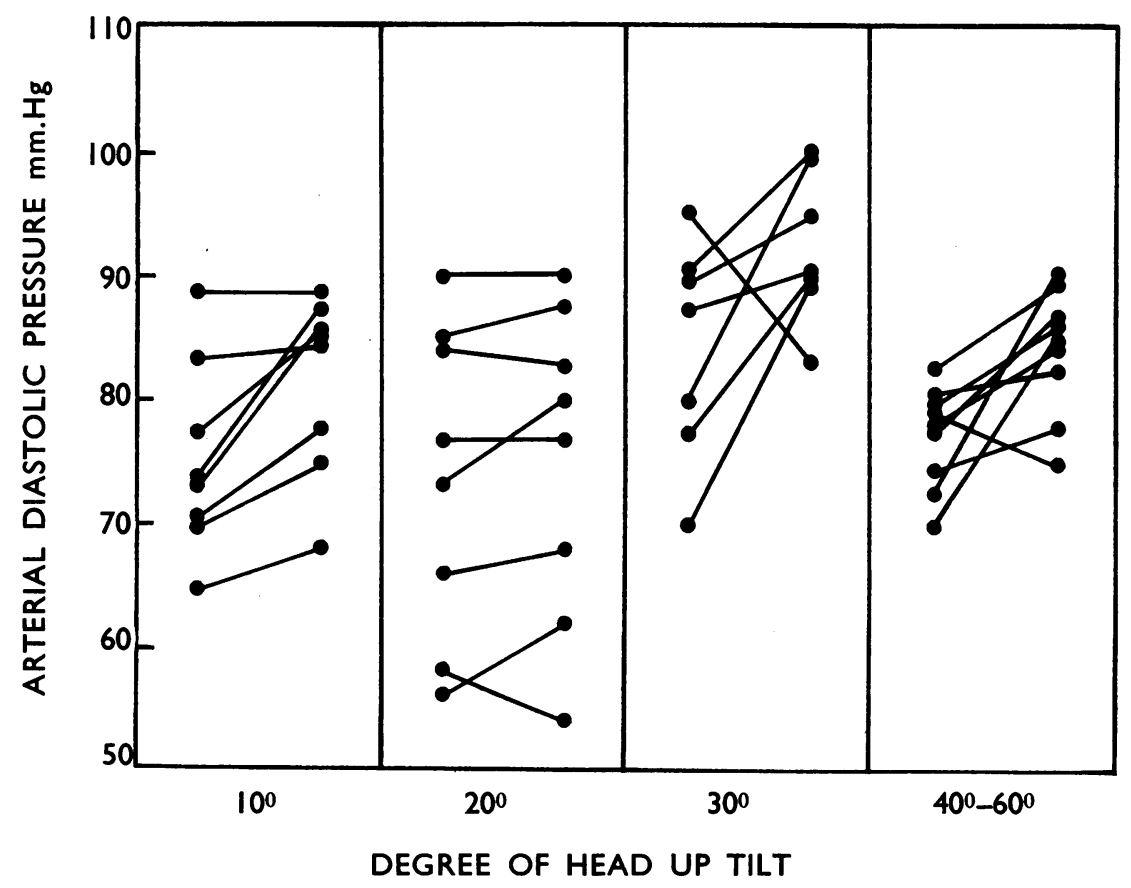

FIG. 5.-Change in arterial diastolic pressure following different degrees of head-up tilt. 


\section{Discussion}

Several studies on the changes of cardiac output associated with postural changes in normal man have been made and they show surprisingly close agreement even though they were done at different positions of tilt. Compared with recumbency the average cardiac outputs were 19 per cent and 23 per cent less (Coe et al., 1950; Stead et al., 1945) in subjects who were tilted from the horizontal to $30^{\circ}$ and $70^{\circ}$, respectively. In two other studies where the subjects first lay flat and then stood relaxed, the fall in average cardiac output was 19 per cent and 20 per cent (Wang et al., 1960; Chapman et al., 1960).

The present investigation adds to the earlier results in that the effects of the length of time in the tilt position, the degree of tilt, and the changes associated with returning to the recumbent position after tilting have been studied.

The results show that a fall in cardiac output occurred during the first 7 minutes of tilting to the head-up position and then became stable and did not change during the remaining 15 minutes of the tilt period.

When compared with the pre-tilt recumbent values, the average cardiac outputs at $10^{\circ}, 20^{\circ}$, and between $30^{\circ}$ and $60^{\circ}$ were decreased 5,17 , and 19 per cent, respectively, and it may be concluded from these data and from those of the previous studies that the decrease in cardiac output in normal man, associated with passive tilt to an upright position, tends to reach its maximum at $20^{\circ}$ with little further decrease between $20^{\circ}$ and $90^{\circ}$, and that most of the decrease occurs between $10^{\circ}$ and $20^{\circ}$. The change from recumbency to upright positions is accompanied by a partial redistribution of blood volume to the dependent parts of the body, and it is generally accepted that this is the cause of the decrease of cardiac output, since it is associated with a fall of atrial pressure and slower diastolic filling of the ventricles (Sjöstrand, 1952; Sieker and Gauer, 1957). But we have shown here that cardiac output does not significantly change after the $20^{\circ}$ position, even though the hydrostatic force which causes the peripheral pooling does increase considerably on further tilting. One possible explanation for the stabilization of cardiac output after $20^{\circ}$ of tilt is that the redistribution of blood volume is effectively resisted to approximately that angle, after which the counteracting effects of venous tone give way and allow nearly maximal peripheral pooling to occur. Another possible explanation is that there may indeed be continued pooling at larger angles of tilt, but the increase of heart rate which begins and gradually increases after $20^{\circ}$, and the probable simultaneous augmentation of myocardial contractility (also caused by a reflex increase of sympathetic nervous system activity) prevent the further decrease of cardiac output (Sarnoff $e t$ al., 1960).

The data in Table I show the difference between the time necessary for the cardiac output to decrease on changing from recumbency to an upright position and for it to increase to its pre-tilt control value after return to the recumbent position. The decrease in cardiac output occurred within 7 minutes after tilting to a head-up position, but even between 11 and 25 minutes after the return to recumbency, the average cardiac output was still 13 per cent below the pre-tilt control value.

It is well established that a change from recumbency to the vertical is accompanied by an increase in heart rate and diastolic pressure with little or no increase in systolic pressure (Hellebrandt and Franseen, 1943). These changes occurred in our series in the subjects tilted to positions between $40^{\circ}$ and $60^{\circ}$. On the other hand, the changes of heart rate and diastolic pressures were not correlated with the magnitude of the fall in cardiac output when subjects were tilted to lesser angles. Although the cardiac output was nearly maximally decreased at $20^{\circ}$ tilt the average heart rate did not significantly increase until the $30^{\circ}$ position and then continued to increase with further tilting. The diastolic pressure, however, was increased at $10^{\circ}$ of tilt. It may well be that the reflexes associated with control of diastolic pressure are more active at lesser degrees of tilt than those responsible for the increase in cardiac rate.

\section{SUMMARY}

The effects of different angles of head-up tilting on cardiac output, heart rate, and arterial blood pressure have been studied. The results show that when normal subjects are placed at head-up tilt positions of either $10^{\circ}, 20^{\circ}, 30^{\circ}, 40^{\circ}, 55^{\circ}$, or $60^{\circ}$, the major change of cardiac output occurs between $10^{\circ}$ and $20^{\circ}$ and there is little change on further tilting to $60^{\circ}$. On return to recumbency, there is a delay of at least 11 to 25 minutes before the cardiac output reaches the level recorded before tilting. The changes in heart rate and systemic blood pressure do not correlate with the magnitude of the decrease in cardiac output. Although the cardiac output does not fall further when the subject is tilted beyond $20^{\circ}$, the stroke output continues to fall up to $60^{\circ}$.

\section{REFERENCES}

Chapman, C. B., Fisher, J. N., and Sproule, B. J. (1960). Behavior of stroke volume at rest and during exercise in human beings. f. clin. Invest., 39, 1208. 
Coe, W. S., Best, M. M., and Lawson, H. C. (1950). The effect of posture and of hypoxia on cardiac output in the normal human subject. Surg. Forum, pages 617-619. Saunders, Philadelphia.

Gabe, I. T., Tuckman, J., and Shillingford, J. P. (1962). Determination of relative changes in cardiac output from noncalibrated earpiece dye-dilution curves. Circulat. Res., 11, 405.

Hellebrandt, F. A., and Franseen, E. B. (1943). Physiological study of the vertical stance of man. Physiol. Rev., 23, 220.

McMichael, J., and Sharpey-Schafer, E. P. (1944). Cardiac output in man by a direct Fick method: effects of posture, venous pressure changes, atropine, and adrenaline. Brit. Heart f., 6, 33.

Reeves, J. T., Grover, R. F., Blount, S. G., Jr., and Filley, F. G. (1961). Cardiac output response to standing and treadmill walking. f. appl. Physiol., 16, 283.
Sarnoff, S. J., Gilmore, J. P., Brockman, S. K., Mitchell, J. H., and Linden, R. J. (1960). Regulation of ventricular contraction by the carotid sinus: its effect on atrial and ventricular dynamics. Circulat. Res., 8, 1123.

Sieker, H. O., and Gauer O. H. (1957). A study of postural effects on pressure relationships in the venous circulation. Clin. Res. Proc., 5, 102.

Sjöstrand, T. (1952). The regulation of the blood distribution in man. Acta physiol. scand., 26, 312.

Stead, E. A., Jr., Warren, J. V., Merrill, A. J., and Brannon, E. S. (1945). The cardiac output in male subjects as measured by the technique of right atrial catheterization. Normal values with observations on the effect of anxiety and tilting. $\mathcal{F}$. clin. Invest., 24, 326.

Wang, J., Marshall, R. J., and Shepherd, J. T. (1960). The effect of changes in posture and of graded exercise on stroke volume in man. $\mathcal{F}$. clin. Invest., 39, 1051. 